\title{
Optimizing Evaluation of Patients with Low-to-Intermediate- Risk Acute Chest Pain: A Randomized Study Comparing Stress Myocardial Perfusion Tomography Incorporating Stress-Only Imaging Versus Cardiac CT
}

\author{
Faisal Nabi ${ }^{1}$, Mahwash Kassi ${ }^{1}$, Kamil Muhyieddeen ${ }^{2}$, Su Min Chang ${ }^{1}$, Jiaqiong Xu ${ }^{3}$, Leif E. Peterson ${ }^{3}$, Nelda P. Wray ${ }^{4}$, \\ Beverly A. Shirkey ${ }^{4}$, Carol M. Ashton ${ }^{4}$, and John J. Mahmarian ${ }^{1}$ \\ ${ }^{I}$ Department of Cardiology, Houston Methodist DeBakey Heart \& Vascular Center, Houston, Texas; ${ }^{2}$ Division of Cardiology, \\ Department of Medicine, University of California San Francisco, Fresno, California; ${ }^{3}$ Center for Biostatistics, Houston Methodist \\ Research Institute, Houston, Texas; and ${ }^{4}$ Department of Surgery, Center for Outcomes Research, Houston Methodist Research \\ Institute, Houston, Texas
}

The purpose of this study was to determine whether stress myocardial perfusion (SPECT) optimized with stress-only (SO) imaging is comparable to cardiac $\mathrm{CT}$ angiography (CTA) for evaluating patients with acute chest pain (ACP). Methods: This was a prospective randomized observational study in 598 ACP patients who underwent CTA versus SPECT. The primary endpoint was length of hospital stay, and secondary endpoints were test feasibility, time to diagnosis, diagnostic accuracy, radiation exposure, and overall cost. Median follow-up was $6.5 \mathrm{mo}$, with a $3.8 \%$ cardiac event rate defined as death or an acute coronary syndrome. Results: Of 2,994 patients screened, $1,703(56.9 \%)$ were not candidates for CTA because of prior cardiac disease $(41 \%)$ or imaging contraindications (16\%). Time to diagnosis $(8.1 \pm 8.5$ vs. $9.4 \pm 7.4 \mathrm{~h})$ and length of hospital stay (19.7 \pm 27.8 vs. $23.5 \pm 34.4 \mathrm{~h}$ ) were significantly shorter with CTA than with SPECT $(P=0.002)$. However, time to diagnosis ( $7.0 \pm 6.2$ vs. $6.8 \pm$ $5.9 \mathrm{~h}, P=0.20)$, length of stay $(15.5 \pm 17.2$ vs. $16.7 \pm 15.3 \mathrm{~h}, P=$ $0.36)$, and hospital costs $(\$ 4,242 \pm \$ 3,871$ vs. $\$ 4,364 \pm 1781, P=0.86)$ were comparable with CTA versus SO SPECT, respectively. SO was also superior to conventional SPECT regarding all of the above metrics and significantly reduced radiation exposure $(5.5 \pm 4.4$ vs. $12.5 \pm 2.7 \mathrm{mSv}, P<0.0001)$. Conclusion: Stress SPECT when optimized with SO imaging is similar to CTA in time to diagnosis, length of hospital stay, and cost, with improved prognostic accuracy and less radiation exposure. Our results emphasize the importance of SO imaging, particularly in low-intermediate-risk emergency room patients who are a population likely to have a normal test result.

Key Words: single photon tomography; coronary artery calcium; cardiac computed tomography

J Nucl Med 2016; 57:378-384

DOI: 10.2967/jnumed.115.166595

N and cardiac CT angiography (CTA) are both accepted modalities for

For correspondence or reprints contact: John J. Mahmarian, Houston Methodist DeBakey Heart \& Vascular Center, 6550 Fannin St., Suite 677, Houston, TX 77030

E-mail: jmahmarian@HoustonMethodist.org

Published online Dec. 3, 2015.

COPYRIGHT (c) 2016 by the Society of Nuclear Medicine and Molecular Imaging, Inc. evaluating low-to-intermediate-risk patients presenting to the emergency department (ED) with acute chest pain (ACP) of uncertain cardiac etiology $(1,2)$. Recent trials suggest that CTA reduces time to diagnosis and length of hospital stay (LOS) more than SPECT $(3,4)$ or standard of care (SOC) $(5,6)$. Whether CTA reduces radiation exposure or hospital costs is controversial (6). The role of unenhanced CT-derived coronary artery calcium score (CACS) in patient management is also unclear $(7,8)$.

We previously reported the value of stress SPECT in a wide spectrum of patients presenting to the ED with ACP (9). When a stressrest SPECT protocol is used, stress-only (SO) imaging can be performed with avoidance of the rest study if the stress study is normal $(10,11)$. The use of SO imaging may optimize SPECT procedures by reducing time to diagnosis, LOS, radiation exposure, and cost. This imaging protocol was not used in prior studies comparing SPECT with CTA $(3,4)$. Therefore, our primary objective was to assess the relative value of SPECT versus CTA for reducing LOS in patients with ACP in which SO imaging was included within the armamentarium of SPECT protocols. LOS is a widely accepted benchmark in current clinical hospital practice, which may be improved through streamlining imaging protocols and thereby reducing time to diagnosis.

\section{MATERIALS AND METHODS}

\section{Study Population}

This was a single-center, randomized prospective observational study comparing CT with stress SPECT in 598 consecutive patients older than 18 y who were hospitalized under observational status awaiting SPECT for evaluation of ACP. Recruitment was from February 2009 to August 2011. Patients were evaluated for study entry on arrival to the SPECT/CT department. SPECT was performed daily from 7 AM to 5 PM whereas CT was performed only Monday through Friday 7 AM to 5 PM. Therefore, patients were not randomized on weekends. The study was approved by the Institutional Review Board of Houston Methodist Research Institute, and all patients signed an informed consent form. Patients excluded from enrollment are shown in Figure 1.

\section{Stress Gated SPECT Imaging}

SPECT was performed as recommended by the American Society of Nuclear Cardiology (12). A stress study was considered normal if left ventricular myocardial perfusion was homogeneous, cavity size was normal, and ejection fraction was $50 \%$ or greater with normal wall motion (10). All other patients underwent rest imaging. 


\section{Cardiac CT}

Patients had CACS followed by CTA using a 64-slice multidetector scanner (Phillips). CACS was acquired in a standard fashion and calculated using the Agatston method. Studies were reported as normal (CACS $=0)$, or showing minimal $(1-10)$, mild $(11-100)$, moderate (101-400), or severe $(>400)$ calcification (13). Electrocardiography-gated CTA was performed after administration of iodinated contrast (Visapaque; GE Healthcare) at $4-5 \mathrm{~mL} / \mathrm{s}$. Patients were pretreated with $\beta$-blockers and $1(0.4 \mathrm{mg})$ sublingual nitroglycerin. Patients with slow and stable heart rates had prospective acquisitions whereas all others had retrospective studies with or without dose modulation. Significant coronary artery disease (CAD) was defined as greater than $50 \%$ stenosis: $51 \%-69 \%$ (moderate), $70 \%-90 \%$ (severe), and greater than $90 \%$ (subtotal/total occlusion).

\section{Clinical Information Collected During Index Visit}

Clinical information and results of cardiac procedures were obtained by research personnel at hospital discharge. The Thrombolysis in Myocardial Infarction and Framingham risk scores were calculated on the basis of standard criteria (14). Time of hospital admission and discharge were captured from the medical record and time of randomization from the consent form. One of 3 nuclear- and CT-board-certified cardiologists interpreted all studies, with time of final interpretation (i.e., time of diagnosis) corresponding with their automatically captured electronic signature. Study results were available to referring physicians immediately on electronic signature.

\section{Patient Follow-up}

Clinical follow-up was prospectively obtained by telephone interview at predefined intervals of $1 \mathrm{wk}, 1 \mathrm{mo}$, and more than 6 mo. Research personnel asked scripted questions regarding any subsequent ED visits, hospital admissions for ACP or myocardial infarction, and invasive coronary angiography (ICA) or coronary revascularization (CR) procedures. The date and place of all admissions and cardiac procedures were verified by medical record review. One investigator masked to the imaging results adjudicated cardiac events. Vital status was obtained through phone follow-up or the Social Security death index.

\section{Cardiac Events}

Cardiac events were defined as cardiac death and acute coronary syndrome (myocardial infarction or unstable angina) (9). Nonfatal myocardial infarction was defined as a troponin greater than $0.10 \mathrm{ng} / \mathrm{mL}$ associated with ACP or electrocardiogram findings of ischemia (9). Unstable angina was defined as new onset/worsening of ACP at rest, normal troponin levels, and $70 \%$ or more stenosis by ICA (9). Cardiac events were counted once in an individual patient when analyzing overall event rates. However, all cardiac events were recorded and designated as an initial or subsequent event after hospital discharge. Patient outcome was defined by time to first cardiac event or last encounter in those without events.

\section{Radiation Exposure}

SPECT radiation exposure was calculated on the basis of the administered radiotracer dose: $\mathrm{mSv}=\mathrm{MBq} \times 0.0069\left({ }^{99 \mathrm{~m}} \mathrm{Tc}\right)$ and $\mathrm{mSv}=$ $\mathrm{MBq} \times 0.14\left({ }^{201} \mathrm{Tl}\right)(15) . \mathrm{CT}$ radiation exposure was calculated on the basis of total dose-length product $\times 0.014$ (4). In-patient radiation exposure did not include that from ICA.

\section{Statistical Analysis}

The primary endpoint was LOS from time of randomization (i.e., arrival to the imaging department). Secondary endpoints were time to diagnosis from randomization (which comprised study acquisition and physician interpretation times), hospital costs, patient outcome, and radiation exposure. Subset analysis was performed to assess primary and secondary endpoints in patients who underwent SO imaging versus CTA or conventional stress-rest SPECT. Patient outcome based on CACS results was also determined. Baseline characteristics are presented as mean \pm SD for continuous variables and number/percentage for categoric variables. The $\chi^{2}$ or Fisher exact test for categoric variables and $t$ test for continuous variables compared differences between SPECT and CTA groups. SPECT/CTA imaging protocols, radiation exposures, and subsequent resource use were summarized.

Accuracy and 95\% confidence intervals of SPECT, CTA, and CACS for predicting events were calculated with $P$ values based on the Fisher exact test. Kaplan-Meier survival curves displayed cardiac events based on SPECT and CTA results with comparison using the log-rank test. Time to diagnosis and LOS were summarized as mean \pm SD by

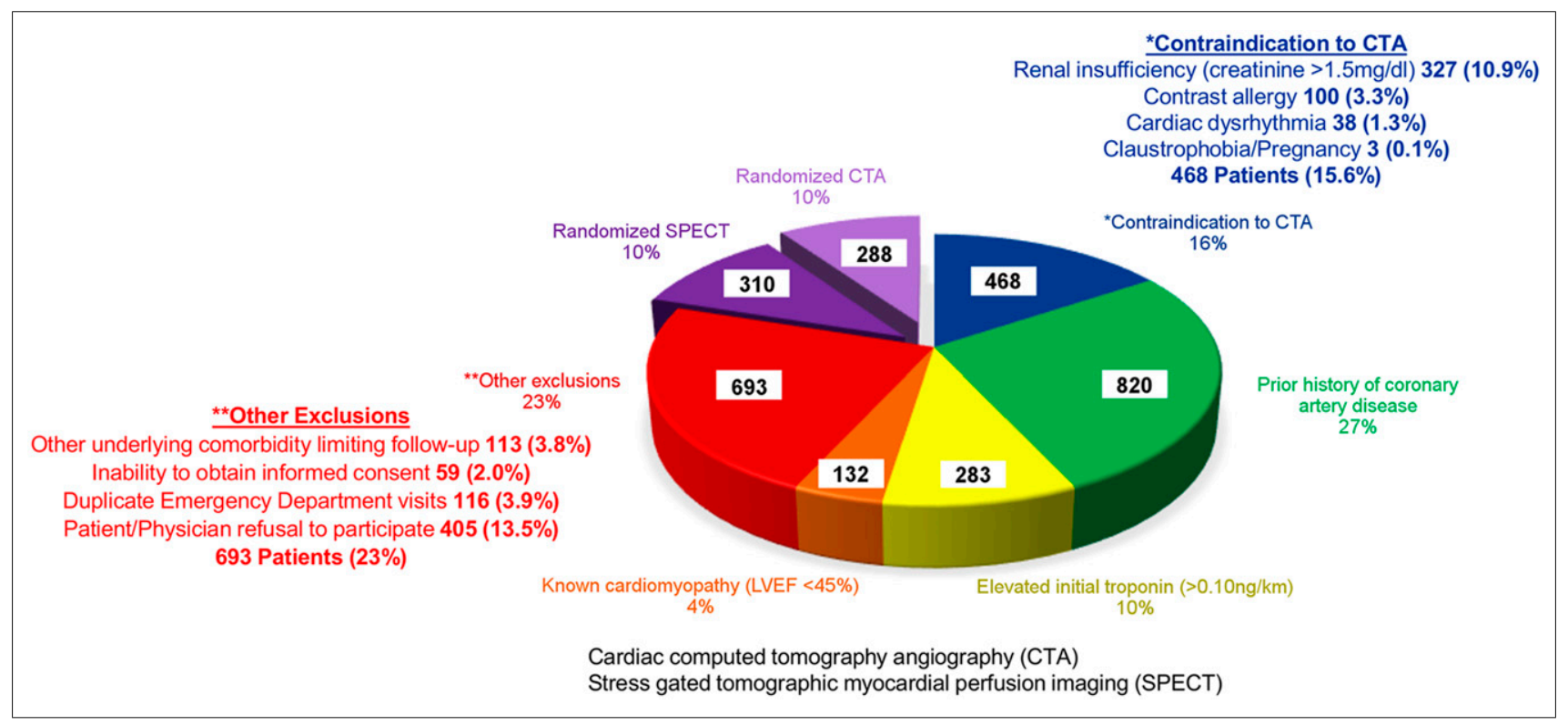

FIGURE 1. Patients screened and excluded from study entry. 
TABLE 1

Baseline Characteristics

\begin{tabular}{|c|c|c|c|c|}
\hline Characteristic & Total $(n=598)$ & $\operatorname{SPECT}(n=310)$ & CTA $(n=288)$ & $P$ \\
\hline Mean age $\pm S D$ & $53.2 \pm 12.2$ & $52.6 \pm 11.9$ & $53.9 \pm 12.5$ & 0.18 \\
\hline Female sex & $332(56.1)$ & $174(56.1)$ & 158 (54.9) & 0.76 \\
\hline Race & & & & 0.05 \\
\hline Black & $178(29.8)$ & 101 (32.6) & $77(26.7)$ & \\
\hline White & 334 (55.9) & $172(55.5)$ & $162(56.3)$ & \\
\hline Hispanic & 69 (11.5) & $30(9.6)$ & $39(13.5)$ & \\
\hline Asian & $8(1.3)$ & $1(0.3)$ & $7(2.4)$ & \\
\hline Other & $9(1.5)$ & $6(1.9)$ & $3(1.1)$ & \\
\hline Body mass index & $31.2 \pm 8.3$ & $31.8 \pm 9.1$ & $30.5 \pm 7.4$ & 0.05 \\
\hline Risk factors & & $5(1,10)$ & $4(2,10)$ & 0.60 \\
\hline Hypertension & $301(50.3)$ & $157(50.7)$ & $144(50.0)$ & 0.88 \\
\hline Diabetes mellitus & $90(15.1)$ & $48(15.4)$ & 42 (14.6) & 0.76 \\
\hline Dyslipidemia & $228(38.1)$ & $115(37.1)$ & $113(39.2)$ & 0.59 \\
\hline Current smoker & $162(27.1)$ & $85(27.2)$ & 77 (26.4) & 0.98 \\
\hline Family history of CAD & $137(22.9)$ & $66(21.3)$ & $71(24.7)$ & 0.33 \\
\hline Acetylsalicylic acid within past $7 \mathrm{~d}$ & $184(30.8)$ & $97(31.3)$ & $87(30.2)$ & 0.78 \\
\hline Severe angina last $24 \mathrm{~h}$ & $32(5.4)$ & $14(4.5)$ & $18(6.3)$ & 0.35 \\
\hline Thrombolysis in Myocardial Infarction risk score & & & & 0.67 \\
\hline 0 & $315(52.7)$ & $170(54.8)$ & $145(50.4)$ & \\
\hline 1 & $183(30.6)$ & $90(29.0)$ & $93(32.3)$ & \\
\hline 2 & $86(14.4)$ & $44(14.2)$ & $42(14.6)$ & \\
\hline 3 & $14(2.3)$ & $6(1.9)$ & $8(2.8)$ & \\
\hline Framingham risk score & & & & 0.95 \\
\hline $0 \%-10 \%$ (low) & $458(76.6)$ & $239(77.1)$ & $219(76.0)$ & \\
\hline $11 \%-20 \%$ (intermediate) & 113 (18.9) & $57(18.4)$ & $56(19.4)$ & \\
\hline$>20 \%$ (high) & $27(4.5)$ & $14(4.5)$ & $13(4.5)$ & \\
\hline
\end{tabular}

Data are mean \pm SD for continuous variables; medians, with interquartile ranges in parentheses if continuous variables were skewed; and numbers, with percentages in parentheses for categoric variables.

randomized group and in the subgroups with normal CTA and SO SPECT. Cumulative density distributions for time to diagnosis and LOS were generated and plotted for SPECT, CT, and SO groups with differences tested using the Wilcoxon rank-sum test. Hospital costs were derived from billing records spanning ED admission to hospital discharge. Costs were estimated from total billed charges multiplied by Medicare cost-to-charge ratio for the year in which the patient received care. Analyses were performed with STATA (version 13; StataCorp LP). Significance was defined as a 2 -tailed $P$ value of less than 0.05 .

\section{RESULTS}

\section{Study Population and Imaging Feasibility (Fig. 1)}

A total of 2,994 consecutive patients over 29 mo were admitted with ACP, of whom 1,703 (56.9\%) were not candidates for CTA because of known cardiovascular disease $(n=$ 1,235 or $41.3 \%$ ) or a contraindication to imaging $(n=468$ or $15.6 \%)$. Of 1,003 remaining eligible patients, 405 (13.5\%) refused participation. The final cohort consisted of 310 patients randomized to SPECT and 288 to a CT strategy. Five patients withdrew after signing consent, and 4 randomized to CTA crossed over to SPECT.

\section{Baseline Characteristics (Table 1)}

Baseline patient characteristics in each randomized strategy were comparable. Although most had a low Thrombolysis in Myocardial Infarction risk score, one-fourth had an intermediate or high Framingham risk score.

\section{Time to Diagnosis and Length of Stay (Figs. 2 and 3)}

Mean times from admission to randomization were comparable in the CT versus SPECT strategy (11.6 \pm 7.8 vs. $12.4 \pm 8.9 \mathrm{~h}, P=0.18)$. Mean time to diagnosis $(8.1 \pm 8.5$ vs. $9.4 \pm 7.4 \mathrm{~h}, P=0.0002)$ and LOS $(19.7 \pm 27.8$ vs. $23.5 \pm 34.4 \mathrm{~h}, P=0.002)$ were significantly shorter with CTA versus SPECT (Fig. 2) as were median values (Fig. 3).

In a prespecified subanalysis in which we compared patients who had a normal CTA versus SO SPECT, time to diagnosis $(7.0 \pm 6.2$ vs. $6.8 \pm 5.9 \mathrm{~h}, P=0.20)$ and $\operatorname{LOS}(15.5 \pm 17.2$ vs. $16.7 \pm 15.3 \mathrm{~h}$, $P=0.36$ ) were comparable for CTA versus SPECT (Fig. 2). Median time to diagnosis and LOS were also comparable in patients who underwent CTA versus SO SPECT (Fig. 3). Mean time to diagnosis 


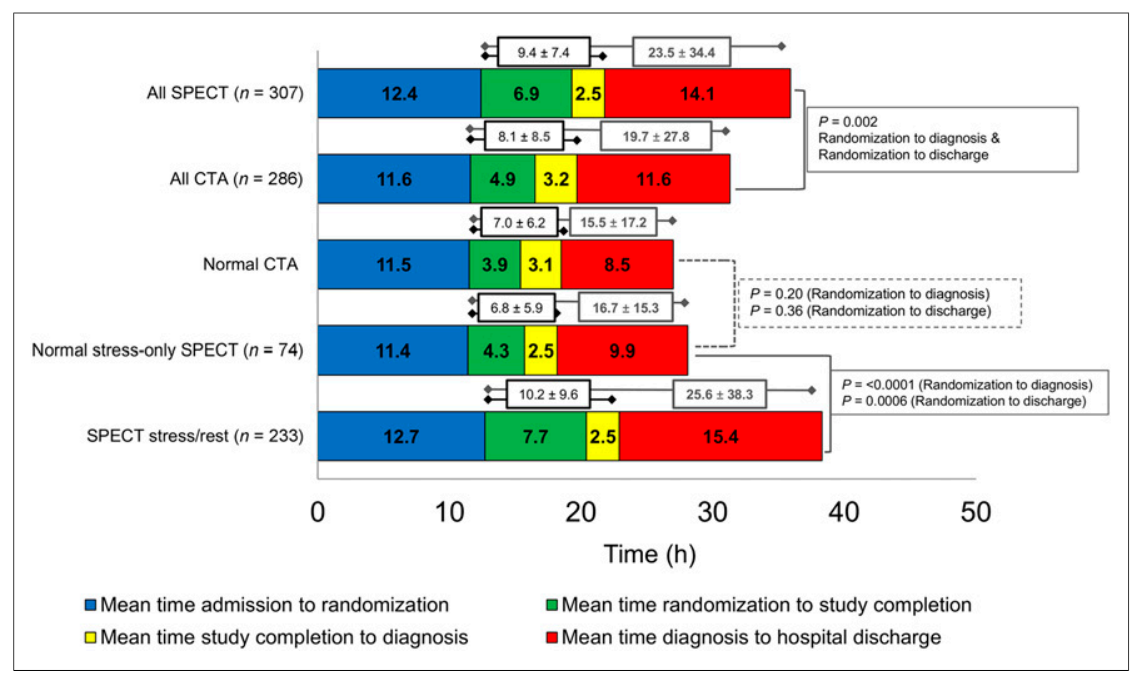

FIGURE 2. Timeline in hours from admission to hospital discharge in randomized groups. There were no significant differences from randomization to diagnosis or hospital discharge in patients who underwent SO SPECT versus a normal CTA.

$(6.8 \pm 5.9$ vs. $10.2 \pm 9.6 \mathrm{~h}, P<0.0001)$ and $\operatorname{LOS}(16.7 \pm 15.3$ vs. $25.6 \pm 38.3 \mathrm{~h}, P<0.0006)$ were significantly shorter for $\mathrm{SO}$ versus conventional SPECT, respectively (Fig. 2).

\section{SPECT/CT Protocols and Radiation Exposure (Table 2)}

Most patients randomized to SPECT had pharmacologic stress testing ( 223 or $73 \%$ ) with adenosine ( 74 or $24 \%$ ), regadenoson (148 or $48 \%$ ), or dobutamine (1 or $0.3 \%$ ). Eighty-four (27\%) had treadmill exercise. Most patients $(96 \%)$ received ${ }^{99 \mathrm{~m} T c-b a s e d ~ r a d i o t r a c e r s, ~ a n d ~}$ 74 (24\%) underwent SO imaging. The mean ${ }^{99 \mathrm{~m}} \mathrm{Tc}$ dose was $1,454 \pm$ $629 \mathrm{MBq}$, which varied depending on imaging protocol. The mean radiation exposure was $10.9 \pm 4.4 \mathrm{mSv}$, which was significantly lower with SO $(5.5 \pm 4.4 \mathrm{mSv})$ than with conventional $(12.5 \pm 2.7 \mathrm{mSv}$, $P<0.0001)$ SPECT.

With CT, prospective acquisitions were performed in 54 patients, with retrospective helical acquisitions (with/without dose modulation) in all others. Overall radiation exposure was $12.7 \pm 4.9 \mathrm{mSv}$.

Radiation exposure was significantly lower in patients who underwent SPECT $(10.9 \pm 4.4 \mathrm{mSv})$ versus CT $(12.7 \pm 4.9 \mathrm{mSv}$, $P<0.0001)$. Total inpatient diagnostic radiation exposure was
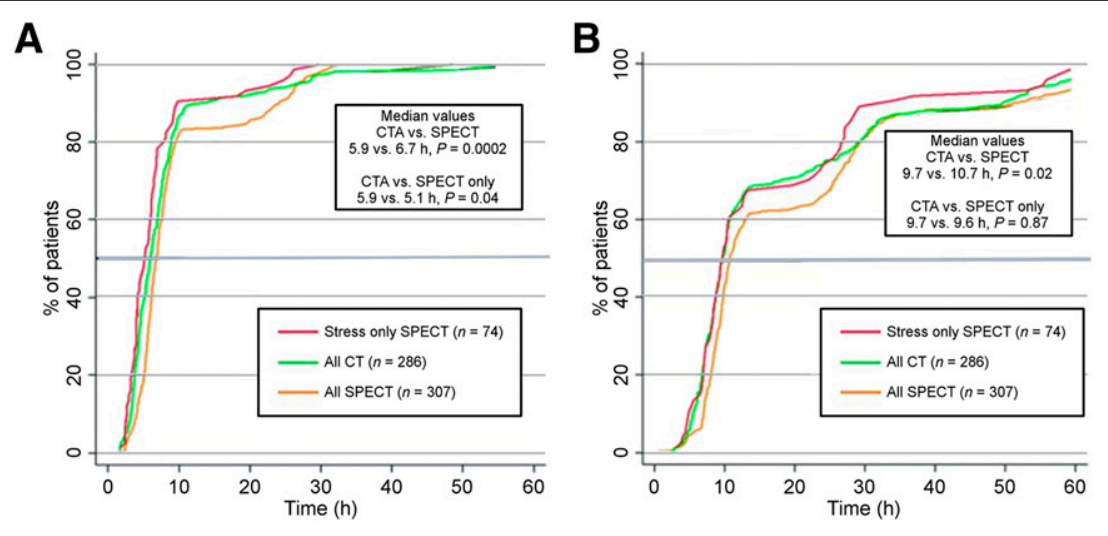

FIGURE 3. Time to diagnosis and hospital discharge in randomized groups. Cumulative frequency from randomization to final diagnosis (A) and hospital discharge (B) for CTA, overall SPECT, and SO groups. Horizontal line is at 50th percentile. also lower in patients randomized to SPECT $(11.0 \pm 4.6 \mathrm{mSv})$ versus CT $(13.3 \pm 5.8 \mathrm{mSv}, P<0.0001)$, for which 4 patients underwent subsequent CTA in the SPECT group and 14 underwent subsequent SPECT in the CT group.

\section{Cardiac Events and Resource Use in Randomized Groups (Supplemental Table 1)}

Follow-up was complete in $98 \%$ (300/ $307)$ and $99 \%(283 / 286)$ of patients randomized to SPECT and CT, respectively $(P=0.34)$. Median follow-up was comparable in the SPECT (6.4; range, 5.9-19.1 mo) and CT (6.5; range, 5.8-23.5 mo) strategies $(P=0.47)$. Twenty-three cardiac events occurred in 22 patients (event rate, $3.8 \%$ ), 18 events during the index admission and 5 in follow-up. All events were unstable angina, except for 1 myocardial infarction. Event rates were similar between strategies ( 9 or $3.0 \%$ SPECT; 13 or $4.6 \% \mathrm{CT} ; P=0.39$ ). There were no significant differences in outpatient, ED, or inpatient cardiac evaluations in the 2 randomized groups or in additional cardiac testing after hospital discharge (Supplemental Table 1; supplemental materials are available at http://jnm.snmjournals.org).

\section{Diagnostic Accuracy: SPECT, CTA, and CACS Results (Fig. 4; Supplemental Table 2)}

SPECT findings were normal in 296 (96.4\%) patients, of whom $14(4.7 \%)$ had ICA during the initial hospitalization $(n=4)$ or after hospital discharge $(n=10)$, with 2 having CR (event rate, $0.7 \%)$. In the $11(3.6 \%)$ patients with abnormal SPECT findings, 9 had ICA during initial hospitalization $(n=8)$ or in follow-up $(n=1)$ and 7 of 9 had severe $(>70 \%$ ) stenosis, with CR in 5. SPECT accuracy for predicting events is shown in Supplemental Table 2.

A normal CTA finding or one with nonobstructive CAD was seen in $245(86.9 \%)$ patients, of whom $7(2.9 \%)$ had ICA during initial hospitalization $(n=2)$ or after hospital discharge $(n=5)$. Only 2 $(0.8 \%)$ had a subsequent cardiac event. In the 33 patients with significant $\mathrm{CAD}, 24$ had single-vessel and 9 multivessel CAD. Nine of 13 patients with ICA had CR. In the other 20 patients, 18 had single-vessel CAD with only moderate $(51 \%-69 \%)$ stenosis and no subsequent events. Event rates increased with CAD extent: normal and nonobstructive, 0.8\%; 1-vessel, 16.7\%; 2-vessel, 71.4\%; and 3-vessel, $100 \%(P<0.001)$. CTA accuracy for predicting events is shown in Supplemental Table 2. Event rates were similarly low in patients with normal SPECT and CTA findings but significantly higher in those with an abnormal SPECT versus CTA $(P=0.03)$ (Fig. 4).

A CACS of 0 was observed in $152(53.9 \%)$ patients, but $76(27 \%)$ had a mild, $28(10 \%)$ moderate, and $26(9.2 \%)$ severe CACS. Although no patient with a CACS of 0 had significant $(>50 \%) \mathrm{CAD}$, this increased with CACS severity: $6.6 \%$ (CACS, 1- 
TABLE 2

SPECT/CT Imaging Protocols and Associated Radiation Exposure

\begin{tabular}{|c|c|c|c|}
\hline Protocol & $n$ & Radiotracer dose (MBq) & Radiation exposure (mSv) \\
\hline \multicolumn{4}{|l|}{ SPECT } \\
\hline Rest-stress & $124(40.4)$ & $1,698 \pm 148$ & $11.7 \pm 1.0$ \\
\hline Stress-rest & $74(24.1)$ & $1,480 \pm 507$ & $12.5 \pm 3.0$ \\
\hline 2-day & $35(11.4)$ & $2,253 \pm 570$ & $15.5 \pm 3.9$ \\
\hline Stress-only & $74(24.1)$ & $644 \pm 374$ & $5.5 \pm 4.4$ \\
\hline Stress-rest or rest-stress & $233(75.9)$ & $1,713 \pm 448^{\star}$ & $12.5 \pm 2.7^{*}$ \\
\hline Overall, 49 of 307 (16\%) patients received $<5 \mathrm{mSv}$ & & $1,454 \pm 629$ & $10.9 \pm 4.4$ \\
\hline \multicolumn{4}{|l|}{ CTA } \\
\hline Retrospective & $126(44.7)$ & & $16.4 \pm 3.3$ \\
\hline Retrospective with dose modulation & $102(36.2)$ & & $12.0 \pm 2.4$ \\
\hline Prospective & $54(19.1)$ & & $5.2 \pm 0.6$ \\
\hline Overall, 20 of $282(7.1 \%)$ patients received $<5 \mathrm{mSv}$ & & & $12.7 \pm 4.9$ \\
\hline $\begin{array}{l}{ }^{\star} P<0.0001 \text { stress-only vs. stress-rest. } \\
\text { Data in parentheses are percentages. }\end{array}$ & & & \\
\hline
\end{tabular}

100), $42 \%$ (CACS, 101-400), and 64\% (CACS, >400). No patient with a CACS of 0 had a cardiac event, but this increased to $10.2 \%$ for those with a CACS greater than $0: 4 \%$ for mild, $18 \%$ for moderate, and $20 \%$ for severe CACS $(P<$ $0.001)$. CACS accuracy for predicting events is shown in Supplemental Table 2.

\section{Cost}

The mean cost in the CT versus SPECT strategy was significantly less $(\$ 4,242 \pm \$ 3,871$ vs. $\$ 5,104 \pm \$ 3,703, P=$ 0.006), with a savings of $\$ 862.00 /$ patient. However, there were no significant cost differences when CT was compared with SO SPECT $(\$ 4,242 \pm \$ 3,871$ vs. $\$ 4,364 \pm \$ 1781, P=0.86)$. SO led

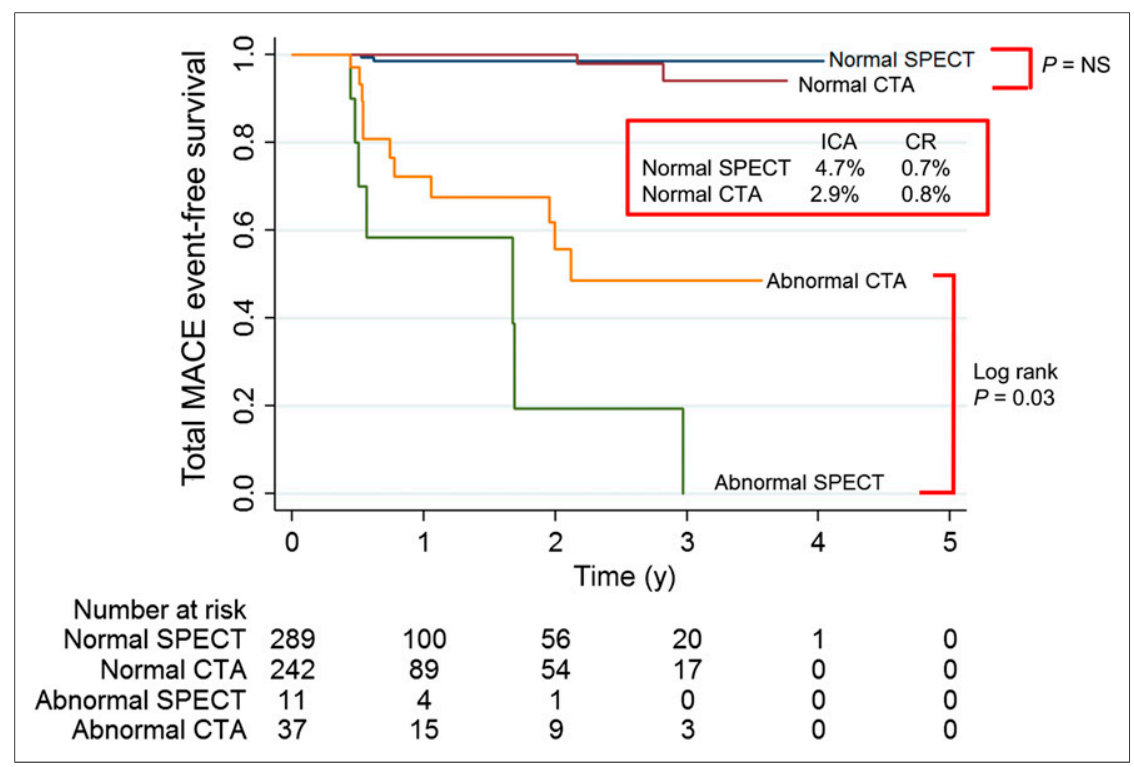

FIGURE 4. Cardiac events based on SPECT and CTA results survival curves show comparably low cardiac event rates in patients with normal SPECT and CTA and low rates of ICA and CR. to a cost savings of $\$ 1,233 /$ patient $(P=0.01)$ versus conventional SPECT.

\section{DISCUSSION}

We studied the relative value of stress SPECT versus CT in lowto-intermediate-risk patients with ACP admitted to a large tertiary care city hospital based on standard metrics including test feasibility, time to diagnosis, LOS, diagnostic accuracy, relative cost, and radiation exposure. A unique aspect of this study was performing SO imaging in patients whenever possible so as to allow comparison not only with CT but also with conventional stress-rest SPECT. Previous studies comparing CTA with SPECT $(3,4)$ did not incorporate SO imaging within the SPECT strategy. All other studies have compared CTA with SOC, with the latter encompassing a heterogeneous assortment of testing algorithms $(5,6,16)$.

Our study not only confirms previous reports but also emphasizes the importance of optimizing SPECT protocols when evaluating patients with ACP so as to promote a patient-centered imaging approach when choosing among various testing modalities. First, our results confirm that many patients who present to the ED with ACP $(57 \%)$ are not candidates for CTA either because of prior history of cardiovascular disease or a contraindication to the procedure $(4,17)$. Conversely, SPECT has few contraindications and is an optimal test in patients with known CAD for whom the main clinical question is the presence and extent of myocardial ischemia $(9,18)$. Second, radiation exposure was higher with CT than SPECT even though most patients $(56 \%)$ underwent radiation-reduction 
strategies with CTA using prospective or dose-modulated retrospective acquisitions. The lower radiation exposure with SPECT was directly attributable to SO imaging, which was performed in approximately $25 \%$ of patients. Third, the overall prognostic accuracy of SPECT $(98 \%)$ was greater than CTA $(91 \%, P<0.0001)$ although both tests were comparable in identifying low-risk patients with a normal test result $(4,9)$. Fourth, as in previous trials, time to diagnosis, LOS, and costs were significantly less with CTA than SPECT $(3,4)$. However, advantages with CTA were lost in patients who underwent SO SPECT. SO imaging was also superior to conventional SPECT regarding the above metrics. Our results illustrate the importance of performing SO imaging whenever possible in low-risk ED patients, of whom most $(>90 \%)$ will have a normal test result. Lastly, our study suggests the potential role of CACS in the ED setting because none of the 54\% patients with a CACS of 0 had significant CAD by CTA or a cardiac event in follow-up.

\section{CTA Versus SPECT in ACP Patients}

Our results confirm previous reports that many patients with ACP (57\%) are not candidates for CTA $(47 \%$ in Coronary Computed Tomographic Angiography for Systematic Triage of Acute Chest Pain Patients to Treatment and 51\% in Rule Out Myocardial Infarction using Computer Assisted Tomography) (4,17). Conversely, SPECT has few contraindications and is an optimal test in patients with known CAD for whom the main clinical question is defining the presence and extent of myocardial ischemia. For example, adenosine SPECT has been shown to safely risk-stratify patients even during acute myocardial infarction (18). There are several randomized studies demonstrating similar diagnostic accuracy, cardiac event rates, and postdischarge resource use with CTA versus SOC (3-6). In 2 studies in which SPECT was the SOC, median times to diagnosis were significantly reduced with CTA (3.4 vs. 15 and 2.9 vs. 6.2 h) (3,4). However, both studies used conventional SPECT, which inevitably led to hours of additional testing. In our study, median time to diagnosis was also significantly less for CTA versus SPECT (5.9 vs. $6.7 \mathrm{~h}, P=0.0002$ ), albeit only a 48-min difference. With SO imaging, time to diagnosis was further reduced to $5.1 \mathrm{~h}$ and with LOS and costs comparable to CTA.

\section{Importance of SO Imaging}

Trials in more than 20,000 patients have demonstrated the feasibility and long-term safety of normal SO versus conventional SPECT $(10,11)$, with marked reductions in radiotracer dose and radiation exposure. Chang et al. reported a mean ${ }^{99 \mathrm{~m}} \mathrm{Tc}$ dose of $788 \mathrm{MBq}$ with SO versus $2,038 \mathrm{MBq}$ with stress-rest imaging. We also reported a significantly lower radiotracer dose (644 \pm 374 vs. $1,713 \pm 448 \mathrm{MBq})$ and radiation exposure $(5.5 \pm 4.4$ vs. $12.5 \pm$ $2.7 \mathrm{mSv}$ ) with SO versus conventional SPECT, respectively. Radiation exposure was less than $5 \mathrm{mSv}$ in $66 \%$ of patients in the SO group, similar to the $60 \%$ incidence previously reported (10). Our low exposure rates were achieved using conventional $\gamma$-camera technology but may be further reduced to $1-2 \mathrm{mSv}$ when combined with cadmium-zinc telluride SPECT (19) and with 40\%-50\% reductions in occupational exposure (20). To date, only a small minority of nuclear cardiology laboratories in North America and worldwide perform SO imaging (21). We have previously reported its value in patients with known or suspected CAD (10). The current study expands SO imaging to the large cohort of lowintermediate-risk patients admitted annually through the ED with ACP. Our study demonstrates that SO imaging reduces time to diagnosis, decreases LOS, and reduces costs and radiation exposure as compared with conventional SPECT in this population. Although we did not routinely use attenuation correction in our patients, this technique aids interpretation of stress-first studies as being normal (22) and might further increase the percentage of SO studies in an ED population such as ours.

\section{CACS}

CACS may have several advantages over CTA in a low-risk cohort without prior $\mathrm{CAD}$ in that it is a rapid, easily interpretable test that has no contraindications and requires no patient preparation. CACS may also challenge SPECT, which requires additional patient preparation, must be performed in conjunction with a stressor modality through coordination of medical and technical staff, is dependent on radiotracer availability, has higher radiation exposure, and may require considerable expertise for accurate interpretation. Most low-to-intermediaterisk patients $(\sim 55 \%)$ with ACP have a CACS of $0(7,17,23-28)$ in whom there is a low likelihood of abnormal SPECT (0.8\%) (7) or significant $\mathrm{CAD}$ as shown in the current study. Pooled data from 8 studies and ours suggest high sensitivity (96\%; 95\% confidence interval, 92\%-98\%) and negative predictive accuracy $(99.4 \%$; 95\% confidence interval, 99\%-100\%) (7,17,23-28) for excluding ACS with CACS, which is comparable to CTA or SPECT $(3-7,9)$. Further study is needed to determine whether initial testing with CACS might safely avoid subsequent additional testing in patients with a normal result.

This study had limitations. This was a single-center study but included patients similar to those reported in other multicenter trials comparing SPECT with CTA $(3,4)$. Second, although our event rate was low, it was comparable to other studies evaluating patients with CTA (3.8 vs. 4.4\%) (16). Third, most patients were not imaged in the ED but admitted under observational care because neither CTA nor SPECT was available in the evening hours. Although 24-h-perday imaging may be optimal, few hospitals currently provide this level of service. Fourth, all patients had CTA using a 64-detector scanner in a population with a mean body mass index of approximately 31 . All patients randomized to CT also underwent calcium scoring, which added approximately $2 \mathrm{mSv}$ to radiation exposure. We recognize that radiation exposure would have been significantly less if we had used newer-generation CT systems (29) and enrolled thinner patients than our cohort. However, in reality, most facilities still currently use conventional $\gamma$-cameras and 64-detector scanners when performing SPECT and cardiac CT, respectively. The 12.7$\mathrm{mSv}$ radiation exposure we report for CTA is similar to the $12-\mathrm{mSv}$ dose recently reported by the PROMISE investigators in 4,996 patients randomized to CTA from 193 sites in North America (30). In this regard our exposure rates are comparable to current clinical practice.

\section{CONCLUSION}

Stress SPECT when optimized with SO imaging is similar to CTA in time to diagnosis, LOS, and cost, with improved prognostic accuracy and less radiation exposure. Our results emphasize the importance of SO imaging particularly in low-intermediate-risk ED patients who are a population likely to have a normal test result.

\section{DISCLOSURE}

The costs of publication of this article were defrayed in part by the payment of page charges. Therefore, and solely to indicate this fact, this article is hereby marked "advertisement" in accordance with 18 USC section 1734 . No potential conflict of interest relevant to this article was reported. 


\section{REFERENCES}

1. Taylor AJ, Cerqueira M, Hodgson JM, et al. 2010 Appropriate use criteria for cardiac computed tomography. Circulation. 2010;122:e525-e555.

2. Hendel RC, Berman DS, Di Carli MF, et al. 2009 Appropriate use criteria for cardiac radionuclide imaging: a report of the American College of Cardiology Foundation Appropriate Use Criteria Task Force, the American Society of Nuclear Cardiology, the American College of Radiology, the American Heart Association, the American Society of Echocardiography, the Society of Cardiovascular Computed Tomography, the Society for Cardiovascular Magnetic Resonance, and the Society of Nuclear Medicine. J Am Coll Cardiol. 2009;53:2201-2229.

3. Goldstein JA, Gallagher MJ, O'Neill WW, Ross MA, O'Neill BJ, Raff GL. A randomized controlled trial of multi-slice coronary computed tomography for evaluation of acute chest pain. J Am Coll Cardiol. 2007;49:863-871.

4. Goldstein JA, Chinnaiyan KM, Abidov A, et al. The CT-STAT (Coronary Computed Tomographic Angiography for Systematic Triage of Acute Chest Pain Patients to Treatment) trial. J Am Coll Cardiol. 2011;58:1414-1422.

5. Litt HI, Gatsonis C, Snyder B, et al. CT Angiography for safe discharge of patients with possible acute coronary syndromes. N Engl J Med. 2012;366:1393-1403.

6. Hoffmann U, Truong QA, Schoenfeld DA, et al. Coronary CT angiography versus standard evaluation in acute chest pain. N Engl J Med. 2012;367:299-308.

7. Nabi F, Chang SM, Pratt CM, et al. Coronary artery calcium scoring in the emergency department: identifying which patients with chest pain can be safely discharged home. Ann Emerg Med. 2010;56:220-229.

8. Hollander JE. Coronary artery calcium scoring: are the data rock solid? Ann Emerg Med. 2010;56:230-232.

9. Nabi F, Chang SM, Xu J, Gigliotti E, Mahmarian JJ. Assessing risk in acute chest pain: the value of stress myocardial perfusion imaging in patients admitted through the emergency department. J Nucl Cardiol. 2012;19:233-243.

10. Chang SM, Nabi F, Xu S, Raza U, Mahmarian JJ. Normal stress-only versus standard stress/rest myocardial perfusion imaging: similar patient mortality with reduced radiation exposure. J Am Coll Cardiol. 2010;55:221-230.

11. Duvall WL, Wijetunga MN, Klein TM, et al. The prognosis of a normal stressonly Tc-99m myocardial perfusion imaging study. J Nucl Cardiol. 2010;17:370377.

12. Hansen CL, Goldstein RA, Akinboboye OO, et al. Myocardial perfusion and function: single photon emission computed tomography. J Nucl Cardiol. 2007;14:e39-e60.

13. Agatston AS, Janowitz WR, Hildner FJ, Zusmer NR, Viamonte M Jr, Detrano R. Quantification of coronary artery calcium using ultrafast computed tomography. J Am Coll Cardiol. 1990;15:827-832.

14. Antman EM, Cohen M, Bernink PJ, et al. The TIMI risk score for unstable angina/non-ST elevation MI: a method for prognostication and therapeutic decision making. JAMA. 2000;284:835-842.

15. International Commission on Radiological Protection (ICRP). Radiation dose to patients from radiopharmaceuticals: addendum 3 to ICRP publication 53. ICRP publication 106. Ann ICRP. 2008;38:1-197.

16. Hulten E, Pickett C, Bittencourt MS, et al. Outcomes after coronary computed tomography angiography in the emergency department: a systematic review and metaanalysis of randomized, controlled trials. J Am Coll Cardiol. 2013;61:880-892.
17. Hoffmann U, Bamberg F, Chae CU, et al. Coronary computed tomography angiography for early triage of patients with acute chest pain: the ROMICAT (Rule Out Myocardial Infarction using Computer Assisted Tomography) trial. J Am Coll Cardiol. 2009;53:1642-1650.

18. Mahmarian JJ, Shaw LJ, Filipchuk NG, et al. A multinational study to establish the value of early adenosine technetium-99m sestamibi myocardial perfusion imaging in identifying a low-risk group for early discharge after acute myocardial infarction. J Am Coll Cardiol. 2006;48:2448-2457.

19. Duvall WL, Croft LB, Ginsberg ES, et al. Reduced isotope dose and imaging time with a high-efficiency CZT SPECT camera. J Nucl Cardiol. 2011;18:847-857.

20. Duvall WL, Guma KA, Kamen J, et al. Reduction in occupational and patient radiation exposure from myocardial perfusion imaging: impact of stress-only imaging and high-efficiency SPECT camera technology. J Nucl Med. 2013;54: 1251-1257.

21. Einstein AJ, Pascual TNB, Mercuri M, et al. Current worldwide nuclear cardiology practices and radiation exposure: results from the 65 country IAEA Nuclear Cardiology Protocols Cross Sectional Study (INCAPS). Eur Heart J. 2015;36:1689-1696

22. Gemignani AS, Muhlebach SG, Abbott BG, Roye GD, Harrington DT, Arrighi JA. Stress-only or stress/rest myocardial perfusion imaging in patients undergoing evaluation for bariatric surgery. J Nucl Cardiol. 2011;18: 886-892.

23. Laudon DA, Vukov LF, Breen JF, Rumberger JA, Wollan PC, Sheedy PF II. Use of electron-beam computed tomography in the evaluation of chest pain patients in the emergency department. Ann Emerg Med. 1999;33:15-21.

24. McLaughlin VV, Balogh T, Rich S. Utility of electron beam computed tomography to stratify patients presenting to the emergency room with chest pain. Am J Cardiol. 1999;84:327-328.

25. Georgiou D, Budoff MJ, Kaufer E, Kennedy JM, Lu B, Brundage BH. Screening patients with chest pain in the emergency department using electron beam tomography: a follow-up study. J Am Coll Cardiol. 2001;38:105-110.

26. Laudon DA, Behrenbeck TR, Wood CM, et al. Computed tomographic coronary artery calcium assessment for evaluating chest pain in the emergency department: long-term outcome of a prospective blind study. Mayo Clin Proc. 2010;85:314-322.

27. Fernandez-Friera L, Garcia-Alvarez A, Bagheriannejad-Esfahani F, et al. Diagnostic value of coronary artery calcium scoring in low-intermediate risk patients evaluated in the emergency department for acute coronary syndrome. Am J Cardiol. 2011;107:17-23.

28. Chou ET, Zakroysky P, Hayden D, et al. Utility of coronary artery calcium scanning in emergency department evaluation of acute chest pain: the ROMICAT II trial [abstract]. J Am Coll Cardiol. 2013;61:e1109.

29. Chen MY, Shanbhag SM, Arai AE. Submillisievert median radiation dose for coronary angiography with a second generation 320-detector row CT scanner in 107 consecutive patients. Radiology. 2013;267:76-85.

30. Douglas PS, Hoffmann U, Patel MR, et al. Outcomes of anatomical versus functional testing for coronary artery disease. N Engl J Med. 2015;372:12911300 . 